ఠ

\title{
Postpartum depression: psychoneuroimmunological underpinnings and treatment
}

This article was published in the following Dove Press journal:

Neuropsychiatric Disease and Treatment

20 February 2013

Number of times this article has been viewed

\author{
George Anderson' \\ Michael Maes ${ }^{2}$ \\ 'CRC Clincial Research Centre/ \\ Communications, Glasgow, Scotland; \\ ${ }^{2}$ Department of Psychiatry, \\ Chulalongkorn University, Bangkok, \\ Thailand
}

\begin{abstract}
Postpartum depression (PPD) is common, occurring in 10\%-15\% of women. Due to concerns about teratogenicity of medications in the suckling infant, the treatment of PPD has often been restricted to psychotherapy. We review here the biological underpinnings to PPD, suggesting a powerful role for the tryptophan catabolites, indoleamine 2,3-dixoygenase, serotonin, and autoimmunity in mediating the consequences of immuno-inflammation and oxidative and nitrosative stress. It is suggested that the increased inflammatory potential, the decreases in endogenous anti-inflammatory compounds together with decreased omega-3 poly-unsaturated fatty acids, in the postnatal period cause an inflammatory environment. The latter may result in the utilization of peripheral inflammatory products, especially kynurenine, in driving the central processes producing postnatal depression. The pharmacological treatment of PPD is placed in this context, and recommendations for more refined and safer treatments are made, including the better utilization of the antidepressant, and the anti-inflammatory and antioxidant effects of melatonin.
\end{abstract}

Keywords: SSRI, kynurenine, IDO, TDO, melatonin

\section{Introduction}

The prevalence of postpartum depression (PPD) is between $10 \%$ and $15 \%$, although generally thought to be considerably underreported. ${ }^{1}$ A prior history of PPD is the major predictive factor for subsequent occurrence. ${ }^{2,3}$ Other risk factors include antenatal depressive symptoms, prenatal neuroticism, lower social support, lower socioeconomic status, obstetric complications, including preeclampsia, and major life events or stressors during pregnancy. ${ }^{4-6}$ PPD is often not recognized and if left untreated can have devastating consequences on the maternal-infant bond as well as on infant mental, motor, and emotional development, leading to depression, anxiety and behavioral problems in the offspring..$^{7-9}$ The Diagnostic and Statistical Manual of Mental Disorders, 4th edition (DSM-IV) treats PPD as a subcategory of major depressive disorder (MDD), and not as a separate disorder.

\section{Predisposing factors}

The susceptibility to depression, including PPD, is the result of epigenetic, genetic, and stress/environment interactions, including in the very early development of the mother herself. Longer-term follow up of PPD offspring at 16 years of age shows that they are four times more likely to be depressed, ${ }^{10}$ suggesting an inter-generational transfer that increases offspring depression and PPD susceptibility.
Correspondence: George Anderson CRC, Rm 30, 57 Laurel Street, Glasgow GII 7QT, Scotland, UK Tel +44750562 3759

Email anderson.george@rocketmail.com 
During pregnancy, the mother is under high oxidative challenge, and dietary factors that impinge on oxidant status, including a range of vitamins and trace elements, are thought to contribute to the etiology of PPD. Changes in fatty acid composition during pregnancy quickly return to the normal range following parturition However, an increase in the omega- $6 / 3$ ratio increases the risk of $\mathrm{PPD}^{11,12}$ Low maternal omega-3 polyunsaturated fatty acid intake is suggested to contribute to decreased maternal and fetal health, interacting with the serotonin transporter alleles to contribute to PPD. ${ }^{13}$

Decreased pregnancy vitamin $\mathrm{D}$ associates with many risk factors for PPD, including preeclampsia, ${ }^{14}$ suggesting that it will indirectly modulate PPD susceptibility. Maternal obesity and excessive weight gain during pregnancy increase PPD risk $^{15}$ as do alterations in levels of maternal leptin at delivery. ${ }^{16}$ However, maternal obesity is associated with decreased vitamin D and omega-3 as well as increased preeclampsia, suggesting that many obesity effects may be indirect. Such dietary susceptibility factors alter the regulation of oxidant status and immuno-inflammatory activations.

Within 48 hours of parturition, maternal levels of cortisol, estrogen, progesterone, and neurosteroids fall dramatically, which has been suggested to contribute to PPD, ${ }^{17,18}$ perhaps paralleling varying depression sensitivity to hormonal changes in menses and menopause. However, other work suggests that hormonal changes are not the major determinant of PPD, ${ }^{19}$ although a decrease in allopregnanolone is correlated with decreased mood in the "baby blues" period postnatally. ${ }^{20}$ Depression during pregnancy, often associated with sleep disturbance, ${ }^{21}$ increases the risk of PPD. ${ }^{22}$ Of note, the risk factors for depression during pregnancy are very similar to the risk factors for $\mathrm{PPD}^{23}$ suggesting that prenatal and postpartum depression are intimately related.

In the third trimester, plasma oxytocin concentration negatively correlates with the postpartum score on the Edinburgh Postnatal Depression Scale, ${ }^{24}$ leading the authors to suggest that targeting an increase in oxytocin during pregnancy may decrease PPD. Oxytocin has a significant role in preparing the mother for the process of delivery as well as for lactation and maternal behavioral adaptations. Much research in this area is preclinical, but human studies also show a significant role for varying oxytocin levels, in both pregnancy and infant attachment. ${ }^{25,26}$ This is of importance as maternal behavioral adaptations, including emotional attachment to the newborn, are often challenged in PPD, resulting in offspring with higher levels of insecure attachment, driving subsequent behavioral and mood problems. ${ }^{10}$ Decreased oxytocin in adults is also linked to increased anxiety and depression. ${ }^{27}$

To accommodate the placenta and developing fetus, the maternal immuno-inflammatory response in normal pregnancy has to adapt. In part, this is driven by high maternal oxidative challenge during pregnancy and is important in how risk factors increase PPD susceptibility. This overlaps PPD to recent conceptualizations of adult depression as an immuno-inflammatory response to oxidative and nitrosative stress (O\&NS), leading to altered tryptophan catabolism, which drives changes in neuronal activity. Here we review how such a psychoneuroimmunological conceptualization of PPD integrates biological data on course and treatment.

\section{Immunological conceptualizations of depression}

Recent conceptualizations of depression have emphasized the effects of increased kynurenine (kyn) in the induction of depression. The driving of the precursor tryptophan down the kyn pathway and away from serotonin and melatonin production is crucial to lowering serotonin in MDD. Single nucleotide polymorphisms (SNPs) in the serotonin ratelimiting enzyme tryptophan hydroxylase 2 (TPH2) gene are linked to increased depression during pregnancy as well as postnatally. ${ }^{28}$ Depressive and anxiety symptoms in the early postnatal period are also linked to a rise in the kyn/tryptophan ratio, ${ }^{29}$ with increases in the proinflammatory cytokines interleukin (IL)- 6 and tumor necrosis factor (TNF)- $\alpha$ in the cerebral spinal fluid at delivery also evident, indicative of elevated central immuno-inflammatory activity. ${ }^{30}$ However, a decrease in serotonin is not the only driver of a depressed response. As well as active depressant effects arising from tryptophan catabolites (TRYCATs), such as kyn and kynurenic acid (KYNA), a reduction in the effects of melatonin, particularly during pregnancy and the postpartum period when anti oxidant defenses are low will have wider inflammatory and immune consequences.

Increased peripheral kyn will also actively contribute to depression. Sixty percent of central kyn is peripherally derived, being readily transported over the blood-brain barrier, where it is converted to KYNA by astrocytes and to wider kyn pathway products, including the excitotoxic quinolinic acid (QUIN), by microglia. KYNA inhibits the alpha 7 nicotinic acetylcholine receptor ( $\alpha 7 \mathrm{nAChr})$, decreasing glutamate, acetylcholine, and dopamine levels in the cortex, contributing to decreased cortex activity, cognition, and cortex influence in refining affectively driven behaviors. ${ }^{31}$ In rodents, prenatal and postnatal exposure to kyn 
results in cognitive deficits in adulthood. ${ }^{32}$ Proinflammatory cytokine induction of indoleamine 2,3-dioxygenase (IDO) drives the TRYCATs increase. It should be noted that IDO has site-specific effects, being important at the placental interface for the immune tolerance necessary for successful pregnancy, but also producing neuroregulatory products such as KYNA and QUIN. As such, the loss of the anti-inflammatory effects of melatonin will directly contribute to proinflammatory cytokine induction of IDO.

Another major inducer of kyn is tryptophan 2,3-dioxygenase (TDO), which is highly expressed in the liver, but also in astrocytes and some neurons..$^{33}$ TDO knockout in the rodent central nervous system is anxioloytic, increasing both neurogenesis and serotonin, the latter 20-fold, emphasizing the importance of TDO in the regulation of central serotonin. ${ }^{34}$ TDO is induced by cyclic adenosine monophosphate (cAMP) and cortisol, both of which are inhibited by melatonin. ${ }^{35}$ Maternal cortisol levels double over pregnancy, suggesting that some of the variation in kyn, KYNA, and kyn/tryptophan ratio may be driven by cortisol induction of TDO, and not solely IDO. The loss of placental melatonin in the maternal circulation following parturition may then contribute to the inflammatory, cytokine, and oxidant induction of TDO and IDO that drive decreases in serotonin and increased kyn in the postpartum period. ${ }^{29}$

The weeks that separate parturition and the emergence of PPD are often stressful, typically incorporating a brief depressive period of "baby blues." In the animal literature, a series of novel stressors is used to induce depression, framed in the context of chronic unpredictable mild stress (CUMS). The driver of CUMS is an increase in peripherally derived kyn, leading to increased levels of QUIN in the amygdala and striatum, as well as a trend increase of KYNA in the cortex. ${ }^{36,37}$ This generally parallels the changes in depression, where heightened amygdale-driven affective processing occurs at the expense of higher order influences on thoughts and behavior. Interestingly, a pattern of decreased orbital frontal cortex negative feedback on amygdala activity heightens and prolongs amygdala activation, increasing the risk of explosive outbursts, evident in about $40 \%$ of people with MDD. ${ }^{38}$ Given the CUMS inhibition of cortex activity, coupled to heightened amygdala activation, it will be interesting to determine the relevance of cortex KYNA and amygdala QUIN to the common fear in PPD of behaving violently to the infant. This could also suggest an important role for variations in the levels of dopamine D1 receptor activity in the paracapsular cells of the intercalated masses, which surround the amygdala and act as a relay for cortex inhibitory feedback. ${ }^{39}$ Dopamine D1r activation hyperpolarizes the paracapsular cells, preventing cortex inhibitory feedback and prolonging enhanced amygdala activation. Interestingly oxytocin alleles interact with the dopamine response to stress, correlating with measures of anxiety, attachment, and emotional wellbeing in nonpregnant women ${ }^{40}$ suggesting an interaction of amygdala oxytocin with stress responses driving depression and associated aggressive impulses.

Increased QUIN in the anterior cingulate is evident in severe depression, an area of the central nervous system linked to emotional processing. ${ }^{41}$ QUIN is excitatory via the N-methyl-D-aspartate receptor, being excitotoxic at higher concentrations. QUIN is produced by IDO activation, probably by amygdala and striatal microglia as a consequence of CUMS. On the other hand, the increase in cortex KYNA decreases cortex arousal. As such, any CUMS effects in the immediate weeks after parturition will increase the amygdalae-driven affective regulation at the expense of higher order cognitive influences on cognitive and behavioral outputs. The associations of hormonal and neurosteroid decreases following parturition would then be sensitizing the mother to the effects of postpartum stressors, acting similarly to CUMS, in driving motivated depressive outputs. Repeat stress is known to decrease allopregnanolone, ${ }^{42}$ an important regulator of IL-1 $\beta$ modulation of oxytocin in pregnancy. ${ }^{43}$ This is a perspective that emphasizes the importance of the TRYCAT pathways in driving immunological influences on patterned neuronal activity, both centrally and peripherally. ${ }^{31,44}$

Interestingly, a decrease in tryptophan is associated more with physiosomatic (formerly psychosomatic) symptoms, ${ }^{45}$ suggesting that the differentiation of somatization and depression may be important to investigate over pregnancy and PPD. We have previously shown that an increase in the kyn/KYNA ratio is specifically associated with somatization, across different DSM-IV categories, ${ }^{46}$ emphasizing the importance of a more refined understanding of the TRYCAT paths. Changes in nociceptive processing locally and centrally may be relevant in PPD, indicated by the dramatic potentiation of pain reporting following cesarean section in mothers who had a depressive episode during pregnancy. ${ }^{47}$ High reporting of feelings of infection, malaise, and fatigue in PPD, coupled with altered T-helper (Th)-1 and Th-2 cytokines is suggestive of wider alterations in immune response, which will contribute to the subjective stress and nociceptive intolerance. ${ }^{48}$

The decrease in leptin at delivery in women with later PPD may also be associated with TRYCAT regulation. ${ }^{16}$ 
Leptin inhibits cortisol production and glucocorticoid receptor activation, suggesting that it will inhibit cortisol induction of TDO. The maternal cortisol levels in late pregnancy correlate with the cortisol response to stress at 6 and 8 weeks postpartum, ${ }^{49}$ indicating a significant role for regulators of pregnancy cortisol response in determining later stress reactivity. Other studies have found no significant effect of pregnancy cortisol as a risk factor for PPD, but have shown wider dysregulation of the hypothalamic-pituitary-adrenal (HPA) axis, including changes in pregnancy corticotrophin releasing hormone. ${ }^{50} \mathrm{~A}$ caveat to measures of cortisol is the association of abusive experiences in women prone to depression, with subsequent posttraumatic stress showing an attenuated cortisol stress response. ${ }^{51}$ This may be relevant to the decrease in cortisol in the study by Salel and colleagues, ${ }^{52}$ where decreased cortisol was associated with severity of PPD. This highlights the wider developmental influences that come to bear in the etiology of depression, including PPD. That said, the interaction of cortisol with leptin is important, with leptin inhibiting the HPA axis and cortisol increasing leptin in healthy individuals. ${ }^{53}$

Leptin is negatively coupled to the cAMP pathways, decreasing cAMP induction of KYNA and TDO and is a significant immune regulator, ${ }^{54}$ increasing Th-1 responses. ${ }^{55}$ Leptin also has prosurvival and protective effects. However, chronically raised leptin leads to leptin resistance, mediated by increased cAMP pathway activity,${ }^{56}$ suppressing the effects of leptin. It is unknown whether increased cAMP in leptin resistance contributes to TRYCAT regulation, linking obesity with MDD and PPD.

The placenta also produces leptin; with placental levels being raised 12-fold in preeclampsia. In the pineal gland of some animals, leptin increases levels of norepinephrineinduced 2, arylalkylamine $\mathrm{N}$-acetyltransferase, increasing melatonin production. ${ }^{57}$ As to whether variations in placental leptin and leptin resistance modulate placental melatonin levels requires investigation. Overall, this suggests a potential role for variations in leptin to modulate different facets of the immune processes driving PPD.

\section{Postpartum depression and immuno- inflammatory pathways}

A number of immuno-inflammatory changes are associated with the puerperium, correlating to postpartum mood and anxiety. Immune activation in the early puerperium, as indicated by increased IL-6, IL-1 receptor antagonist (RA), and leukemia inhibitory factor receptor, as well as decreased anti-inflammatory Clara cell protein correlates with early postpartum anxiety and depressive symptoms ${ }^{58,59}$ Puerperal IL-6 and IL-1RA are further increased in women with a prior history of depression, ${ }^{60}$ with anxiety and inflammatory responses being more evident in primiparae women versus multiparae ${ }^{61}$ Such immuno-inflammatory, mood, and anxiety increases in the early puerperium correlate with decreased tryptophan and an increase in the kyn/tryptophan ratio, ${ }^{29,62}$ although not all studies show a correlation of decreased tryptophan with early postpartum mood. ${ }^{63}$ As such, available data suggest an association of immuno-inflammatory and TRYCAT changes with mood in the early postpartum blues period, and require investigation as to whether this is a direct link to PPD itself. The data as available clearly indicate the postnatal period as an inflammatory condition, increasing the risk of depression, especially in women with a predisposition to depression.

\section{Autoimmunity}

Autoimmunity is associated with depression including serotonin autoimmunity and thyroid autoimmunity ${ }^{64,65}$ Elevations in thyroid stimulating hormone, linked to thyroid autoimmunity, have been proposed as a parturition measure predicting later PPD. ${ }^{66}$ This would link to the decreased thyroid hormone, evident in PPD, where it negatively correlates with PPD severity. ${ }^{52}$ Serotonin autoimmunity is associated with increased physiosomatic symptoms, including malaise and neurocognitive symptoms, as well as increased serum neopterin and lysozyme, coupled to increased plasma TNF- $\alpha$ and IL-1 in comparison with depressed patients without 5-hydroxytryptamine (5-HT) autoimmunity. ${ }^{64}$ This emphasizes the importance that immuno-inflammatory pathways have in the onset of 5-HT autoimmunity. Increased bacterial translocation significantly interacts with these inflammatory processes, ${ }^{67}$ and may be of relevance in parturition, especially following cesarean section. Fatigue is common in PPD, ${ }^{68}$ which like malaise is associated with autoimmune activity. ${ }^{69}$ The interaction of 5-HT autoimmunity with somatization driven by alterations in specific TRYCATs and overlapping with CUMS-associated changes post-parturition in PPD requires investigation. Such a perspective better incorporates the known changes and risk factors in PPD and may lead to more refined treatment, as shown in the summary figure.

\section{Treatment}

Treatment of PPD has often utilized different forms of psychotherapy, given the concern about pharmacological treatment postpartum on the suckling infant. ${ }^{70}$ Other treatment 


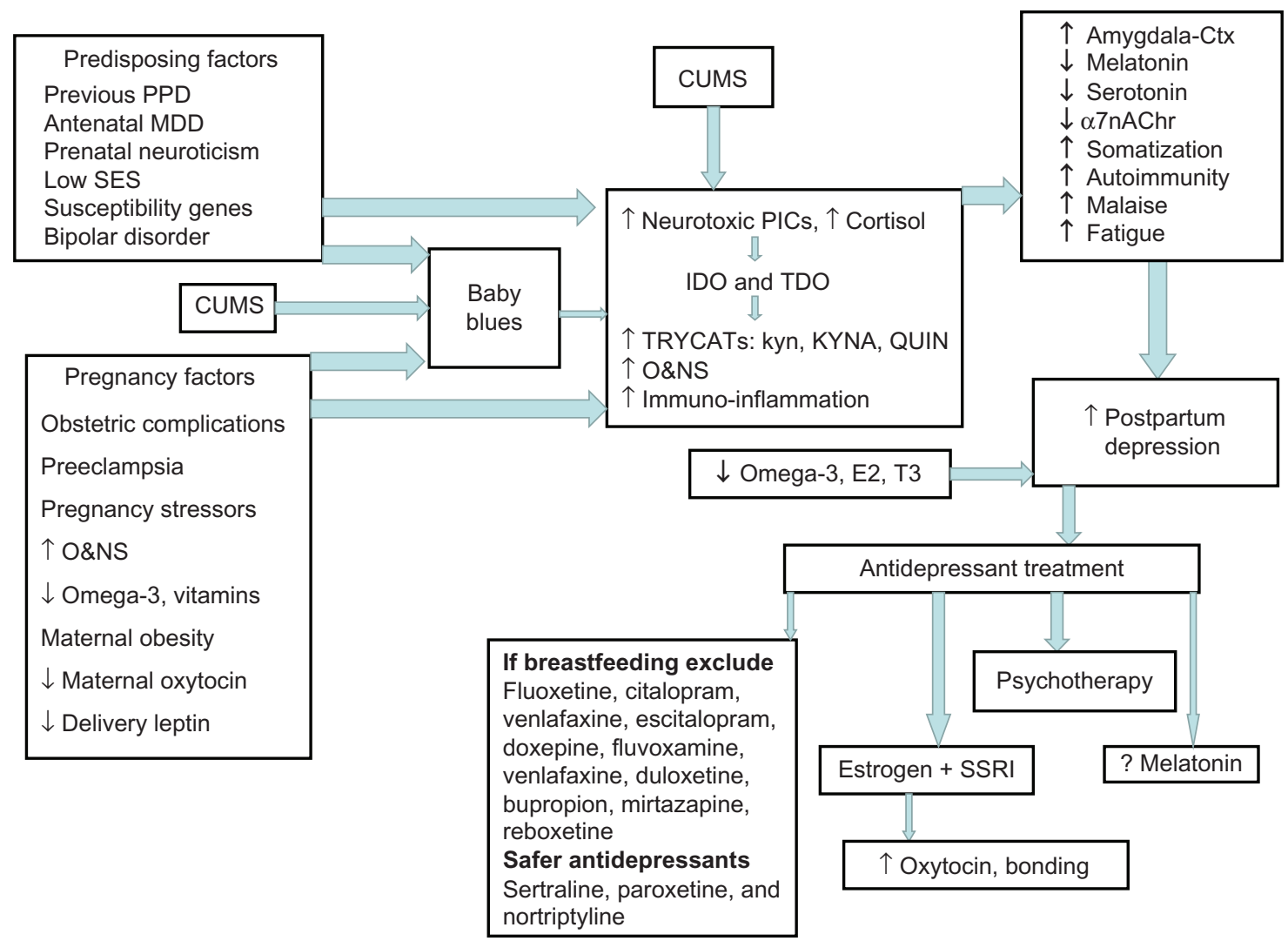

Figure I The predisposing, pregnancy and CUMS factors that contribute to PPD, both directly and via "baby blues" induce TDO and IDO, increasing TRYCATs, including KYNA and QUIN, as well as increasing PICs and O\&NS.

Notes: Such increased immuno-inflammation drives down serotonin, melatonin, and $\alpha 7 n A C h r$, whilst increasing autoimmunity, somatization, and relative amygdalae-cortex activity. Along with decreased omega-3 polyunsaturated fatty acids, this increases PPD. Treatments include psychotherapy and antidepressants. Estrogen can enhance the efficacy of SSRIs, whilst melatonin may provide a safer treatment for both mother and child. Some antidepressants are not recommended if breastfeeding, including those where no relevant data exist.

Abbreviations: $\alpha 7 n A C h r$, alpha 7 nicotinic acetylcholine receptor; CUMS, chronic unpredictable mild stress; E2, estradiol; IDO, indoleamine 2,3-dioxygenase; kyn, kynurenine; KYNA, kynurenic acid; MDD, major depressive disorder; O\&NS, oxidative and nitrosative stress; PIC, proinflammatory cytokine; PPD, postpartum depression; QUIN, quinolinic acid; SES, socioeconomic status; SSRI, selective serotonin reuptake inhibitor; T3, thyroid hormone; TDO, tryptophan 2,3-dioxygenase; TRYCAT, tryptophan catabolite.

approaches are various including preventative exercise, ${ }^{71}$ acupuncture, massage, morning light exposure, and hypnosis. ${ }^{72}$ Here we focus on the role of pharmacological treatments and try to place this in the context of immuneinflammatory pathways, including TRYCATs and O\&NS.

There is a general lack of methodologically sound randomized, double-blind placebo controlled clinical trials of antidepressant treatments in pregnancy as well as in PPD. ${ }^{73}$ Two recent reviews of antidepressants and PPD found only nine studies. ${ }^{70,74}$ Of these, four were randomized, with only two being placebo controlled. ${ }^{75,76}$ The nonrandomized trials tend to show a stronger benefit of antidepressants. However, this has to be tempered by design weakness. In the better controlled studies, selective serotonin reuptake inhibitors (SSRIs) are superior to placebo, ${ }^{76}$ but show no significant advantage when combined with psychotherapy versus psychotherapy alone. The Yonkers et al study produced similar results. ${ }^{75}$
These studies could suggest that psychotherapy would be the more efficacious and safer option. However, a couple of caveats have to be borne in mind. Most of the antidepressant studies in PPD have comprised groups of women with mainly mild to moderate depression. A meta-analysis of the benefits of antidepressants shows that efficacy over placebo increases with severity of depression. ${ }^{77}$ Secondly, although women generally respond better to SSRIs than men, variations in the levels of estrogen postpartum may be interacting with SSRI response, ${ }^{78}$ with low estrogen levels, as evident in $\mathrm{PPD},{ }^{52}$ inhibiting SSRI efficacy. ${ }^{79}$

SSRIs are the most widely used antidepressants in PPD. Systematic reviews on antidepressant use postpartum suggest that sertraline, paroxetine, and nortriptyline are associated with only rare adverse effects in infants and are least likely to show detectable amounts in infant serum. ${ }^{80,81}$ However, detectable amounts of fluoxetine and citalopram have been found in the infant, as with venlafaxine and escitalopram, 
decreasing their use postpartum. ${ }^{82}$ Side effects in infants are found mostly with fluoxetine and citalopram. ${ }^{83}$ The tricyclic doxepine is also not recommended. ${ }^{83,84}$ Particular caution should be used when the baby is premature, of low birth weight, or currently ill, as all these conditions are linked to a decrease in metabolic capacity. A general lack of data on fluvoxamine, venlafaxine, duloxetine, bupropion, mirtazapine, and reboxetine suggests that they are best avoided, pending further studies. As in adult depression more generally, if a patient has shown a positive response to a particular antidepressant in the past, it should be considered first choice, subsequently taking the above into account.

The use of SSRIs, increasing the availability of serotonin, is relevant to our understanding of depression, as outlined above. Preliminary data show that SNPs in IDO interact with antidepressant efficacy, highlighting the role of the IDO pathway in the depression-modifying effects of SSRIs. ${ }^{85}$ An increase in IDO and TDO, driving tryptophan down the kyn pathways and away from serotonin, $\mathrm{N}$-aceytlserotonin, and melatonin production, links with serotonin autoimmunity to suggest that a decrease in serotonin is relevant to depression, including PPD. However, it should be emphasized that the induction of kyn, KYNA, and QUIN is not an incidental sideshow, as these TRYCATs are crucial to the etiology and course of depression. As to whether the utilization of adjunctive melatonin, given its anti-inflammatory and antioxidant effects, would inhibit the O\&NS/inflammation driven changes in TRYCATs in PPD requires investigation. The utilization of melatonin in the treatment of PPD is discussed in more detail below.

Generally, antidepressant treatment would only be commenced with caution, starting with a single treatment at the lowest possible dose. This should be preceded by a careful benefits-risk analysis, with emphasis on the issue of breastfeeding. Breastfeeding may have to be discontinued if dosage is high or multiple pharmacological treatments are used.

\section{Combined treatment}

A combination of psychotherapy and antidepressants is often more efficacious than either alone in the treatment of adult depression. ${ }^{86}$ This is increasingly common in urban areas, but sometimes hard to achieve in rural communities. The utilization of telephone, text, and Internet has proved useful in the absence of direct, personal contact psychotherapy and may be an important point of contact for women with PPD, ${ }^{87}$ including when adjunctive to pharmacotherapy. This requires more investigation.
In a randomized, double-blind, placebo-controlled trial looking at the effects of either sertraline or placebo conjunctive to psychotherapy in the treatment of PPD, there was a trend for additional benefit, but no significant effect of the SSRI. ${ }^{88}$ The Bloch and colleagues study was limited by a small sample size ${ }^{88}$ but like the other well controlled studies, ${ }^{75,76}$ does not provide convincing evidence for the use of SSRIs either in comparison, or adjunctive, to psychotherapy.

\section{Other biological treatments}

A decrease in zinc and magnesium has been shown in PPD. ${ }^{89,90}$ Thiamine (vitamin B1) deficiency increases mouse depression, aggression, confusion and memory impairment, which antidepressants suppress. ${ }^{91}$ In an animal model of PPD, the administration of zinc, magnesium, and thiamine improved depression and anxiety indicants, as well as total antioxidant status, ${ }^{92}$ leading the authors to propose beneficial effects of such a combination in PPD.

Estrogen has been proposed as an antidepressant, including for use in PPD, where it would increase oxytocin levels. ${ }^{93}$ SNPs in the estrogen receptor are associated with increased susceptibility to depression. ${ }^{94}$ As highlighted above, the initial hypogonadism in early $\mathrm{PPD},{ }^{52}$ followed by significant fluctuations in subsequent months, may significantly interact with the efficacy of SSRIs. The use of combinations of estrogen and SSRIs requires careful investigation. Possible adjunctive use of testosterone has also been proposed. Such hormonal treatment is thought to be more beneficial in women with a history of premenstrual depression. ${ }^{93}$ However, this may require a delicate balance with progesterone, which can be problematic, especially when indicants of progesterone intolerance are evident. The role for hormonal treatment of PPD requires further investigation, including as to how hormonal modulations interact with TRYCAT pathways, SSRIs, and oxytocin regulation.

\section{Melatonin and melatonergic medications}

Recently, we proposed the efficacy of melatonin in the treatment of postpartum psychosis and depression in bipolar women. ${ }^{95}$ The tapering down of mood-stabilizers around parturition increases the likelihood of mood dysregulation in the immediate postnatal period, which melatonin may help. Bipolar disorder is by far the major risk factor for postpartum psychosis, but is also a risk factor for PPD. A genetic decrease in melatonin is evident in bipolar disorder. ${ }^{96} \mathrm{~A}$ melatonin receptor SNP is associated with depression risk generally, ${ }^{97}$ suggesting that variations in melatonin will contribute to 
PPD susceptibility. Alterations in melatonin production are evident in depressed pregnant women, as well as in PPD. ${ }^{98}$

Melatonin is a powerful antioxidant, anti-inflammatory, and antinociceptive, and increases mitochondrial oxidative phosphorylation, being generally free of side effects. ${ }^{99}$ The placenta produces melatonin, with levels increasing over pregnancy as the placenta grows. ${ }^{100}$ Melatonin has been used successfully with struggling neonates, with beneficial effects ${ }^{100,101}$ and has been proposed to mediate the beneficial effects of breastfeeding on infantile colic and sleep improvement. ${ }^{102}$

There is growing appreciation of melatonin's antidepressant action, leading to the development of melatonergic-based pharmaceuticals, including agomelatine (a melatonin MT1r and MT2r agonist and serotonin $2 \mathrm{Cr}$ antagonist) and ramelteon (MT1/2r agonist). The efficacy of these melatonergic medications is still to be tested in PPD, although melatonin itself may prove a more effective and safer option. Certainly, the dysregulation in the circadian rhythm and sleep pattern that is common in PPD would be improved, contributing to maternal well-being per se.

Complications in pregnancy, including cesarean section, increase the proinflammatory cytokine TNF- $\alpha$, which inhibits the production of melatonin by the pineal gland for around 2 weeks after parturition. ${ }^{103}$ Levels of postsurgical infection following cesarean section are about $10 \%$ in the UK. ${ }^{104}$ It is unknown as to whether postsurgery infection would modulate the increased risk of PPD following cesarean section or impact on levels of TNF- $\alpha$ and melatonin production. As to whether melatonin would have particular efficacy after cesarean section in improving maternal mood and decreasing subsequent PPD requires investigation.

Maternal prenatal depression increases pain reporting in women who have had a cesarean section. ${ }^{47}$ The greater the severity of acute pain following parturition, irrespective of mode of delivery, increases PPD risk. ${ }^{105}$ As to whether this would have any relevance to increased levels of the kyn/KYNA ratio, which is associated with somatization and differentiates somatization from MDD requires investigation. ${ }^{45,46}$ For some, an increase in somatization, rather than MDD, in pregnancy and in the period between parturition and PPD may occur, involving distinct changes in TRYCATs, including a relative increase in kyn/KYNA ratio or a general increase in both kyn and KYNA. As outlined above, a relative increase in kyn, will be a peripheral source for a CUMS-mediated alteration in central TRYCATs, including amygdalae-driven affective regulation by QUIN. The antinociceptive, anti-inflammatory, and antioxidant effects of melatonin are likely to safely dampen such peripheral drivers of central affective regulation. Factors modulating the regulation of kyn aminotransferase, which mediates the conversion of kyn to KYNA would also be a treatment target for such peripheral to central communication, given that KYNA, unlike kyn, cannot be transferred over the blood-brain barrier. Likewise, further breakdown into other TRYCATs would modulate levels and ratios of specific kyn products. It is unknown whether melatonin would influence this.

KYNA will modulate the central and peripheral immune system, as well as nociception, via the $\alpha 7 \mathrm{nACh}$. Given that agonism at the $\alpha 7 \mathrm{nAChr}$ has antidepressant effects in rodents, when coupled with a subactive dose of SSRI, ${ }^{106}$ suggests that variations in kyn/KYNA ratio, like estrogen, will relevantly modulate SSRI efficacy and doses required. This may have some relevance to the data showing the reemergence of cigarette smoking in women with $\mathrm{PPD}$, who had stopped during pregnancy, ${ }^{107}$ implying a wider and perhaps more direct role of the $\alpha 7 \mathrm{nAChr}$ in the etiology, course, and treatment of PPD. Nicotine/ $\alpha 7 \mathrm{nAChr}$ agonists have antinociceptive effects and would compete with KYNA at the $\alpha 7 \mathrm{nAChr}$, potentiating the effects of SSRIs, whilst allowing KYNA to have continued antinociceptive effects via the direct activation of the GPR35. ${ }^{108}$ Melatonin increases levels and the cellular responsiveness of the $\alpha 7 \mathrm{nAChr} .{ }^{109}$

As to whether these biological processes drive the efficacy of blocking blue light in PPD treatment awaits investigation. Blocking blue light when mothers with PPD awake for middle of the night feeding, prevents melatonin suppression, maintains their circadian rhythm, and hastens recovery from PPD. ${ }^{110}$

Variations in melatonin will also modulate the effects of oxytocin, ${ }^{111,112}$ which when decreased in the third trimester correlates with PPD symptoms. ${ }^{24}$ Melatonin sensitizes myometerial cells to oxytocin, facilitating uterine contractions, ${ }^{111}$ but can also act to inhibit oxytocin release to gonadotropin-releasing hormone. ${ }^{112}$ Given the role of oxytocin, like melatonin, in the regulation of the amygdala and stress-induced cortisol reactivity ${ }^{113,114}$ it is likely oxytocin and melatonin will interact in pregnancy and postpartum to modulate the susceptibility to PPD. Such alterations in the cortisol response and amygdala activity are likely to be driven by changes in IDO and TDO, given the effects of stressinduced inflammatory responses and cortisol respectively, on IDO and TDO induction.

Agomelatine is both an anxioloytic and antidepressant. ${ }^{115}$ Its efficacy in PPD is untested. Ramelteon is likewise untested in PPD. 


\section{Conclusion}

The etiology of PPD, like MDD, is determined by many factors, including in the early development of the mother. Its course is driven by O\&NS and immuno-inflammatory pathways, subsequently regulating TRYCATs, serotonin, and autoimmunity. Its treatment is approached cautiously, with psychotherapy being generally recommended, due to concerns centered on drug transfer during breastfeeding. A better appreciation of its biological underpinnings should lead to a more targeted and safer pharmaceutical intervention. The antidepressant, anti-inflammatory, antioxidant capacities, and proven safety of melatonin are likely to make a significant contribution to the treatment of this disorder, providing better outcomes for both the mother and her child.

\section{Disclosure}

The authors have no disclosures or conflicts of interests to declare.

\section{References}

1. Gaynes BN, Gavin N, Meltzer-Brody S, et al. Perinatal depression: prevalence, screening accuracy, and screening outcomes. Evid Rep Technol Assess (Summ). 2005;119:1-8.

2. Harris B. Biological and hormonal aspects of postpartum depressed moods. Br J Psychiatry. 1994;164(3):288-292.

3. Llewellyn AM, Stowe ZN, Nemeroff CB. Depression during pregnancy and the puerperium. J Clin Psychiatry. 1997;58(Suppl 15):26-32.

4. Hirst KP, Moutier CY. Postpartum major depression. Am Fam Physician. 2010;82(8):926-933.

5. Martin-Santos R, Gelabert E, Subira A, et al. Is neuroticism a risk factor for postpartum depression? Psychol Med. 2012;42:1559-1565.

6. Hoedjes M, Berks D, Vogel I, et al. Postpartum depression after mild and severe preeclampsia. $J$ Womens Health (Larchmt). 2011;20(10): $1535-1542$.

7. Field T. Postpartum depression effects on early interactions, parenting, and safety practices: a review. Infant Behav Dev. 2010;33(1):1-6.

8. Goodman SH, Gotlib IH. Risk for psychopathology in the children of depressed mothers: a developmental model for understanding mechanisms of transmission. Psychol Rev. 1999;106(3):458-490.

9. Walker MJ, Davis C, Al-Sahab B, Tamim H. Reported maternal postpartum depression and risk of childhood psychopathology. Matern Child Health J. In press.

10. Pawlby S, Hay DF, Sharp D, Waters CS, O'Keane V. Antenatal depression predicts depression in adolescent offspring: prospective longitudinal community-based study. J Affect Dis. 2009;113:236-243.

11. De Vriese SR, Christophe AB, Maes M. Fatty acid composition of phospholipids and cholesteryl esters in maternal serum in the early puerperium. Prostaglandins Leukot Essent Fatty Acids. 2003a;68(5):331-335.

12. De Vriese SR, Christophe AB, Maes M. Lowered serum n-3 polyunsaturated fatty acid (PUFA) levels predict the occurrence of postpartum depression: further evidence that lowered n-PUFAs are related to major depression. Life Sci. 2003;73(25):3181-3187.

13. Shapiro GD, Fraser WD, Séguin JR. Emerging risk factors for postpartum depression: serotonin transporter genotype and omega-3 fatty acid status. Can J Psychiatry. 2012;57(11):704-712.

14. Christesen HT, Falkenberg T, Lamont RF, Jørgensen JS. The impact of vitamin D on pregnancy: a systematic review. Acta Obstet Gynecol Scand. 2012;91(12):1357-1367.
15. Milgrom J, Skouteris H, Worotniuk T, Henwood A, Bruce L. The association between ante- and postnatal depressive symptoms and obesity in both mother and child: a systematic review of the literature. Womens Health Issues. 2012;22(3):e319-e328.

16. Skalkidou A, Sylvén SM, Papadopoulos FC, Olovsson M, Larsson A, Sundstrom-Poromaa I. Risk of postpartum depression in association with serum leptin and interleukin-6 levels at delivery: A nested case control study within the UPPSAT cohort. Psychoneuroendocrinology. 2009;34:1329-1337.

17. Bloch M, Meiboom H, Lorberblatt M, Bluvstein I, Aharonov I, Schreiber S. The effect of sertraline add-on to brief dynamic psychotherapy for the treatment of postpartum depression: a randomized, double-blind, placebo-controlled study. J Clin Psychiatry. 2012;73(2): 235-241.

18. Paskova A, Jirak R, Mikesova M, et al. The role of steroids in the development of post-partum mental disorders. Biomed Pap Med Fac Univ Palacky Olomouc Czech Repub. Epub November 12, 2012.

19. Miller LJ. Postpartum depression. JAMA. 2002;287(6):762-765.

20. Nappi RE, Petraglia F, Luisi S, Polatti F, Farina C, Genazzani AR. Serum allopregnanolone in women with postpartum "blues". Obstet Gynecol. 2001;97(1):77-80.

21. D Rheim SK, Bjorvatn BR, Eberhard-Gran M. Insomnia and depressive symptoms in late pregnancy: a population-based study. Behav Sleep Med. 2012;10(3):152-166.

22. Silva R, Jansen K, Souza L, et al. Sociodemographic risk factors of perinatal depression: a cohort study in the public health care system. Rev Bras Psiquiatr. 2012;34(2):143-148.

23. Lancaster CA, Gold KJ, Flynn HA, Yoo H, Marcus SM, Davis MM. Risk factors for depressive symptoms during pregnancy: a systematic review. Am J Obstet Gynecol. 2010;202(1):5-14.

24. Skrundz M, Bolten M, Nast I, Hellhammer DH, Meinlschmidt G. Plasma oyxtocin concentration during pregnancy is associated with development of postpartum depression. Neuropsychopharmacology. 2011;36:1886-1893.

25. Feldman R, Weller A, Zagoory-Sharon O, Levine A. Evidence for a neuroendocrinological foundation of human affiliation: plasma oxytocin levels across pregnancy and the postpartum period predict mother-infant bonding. Psychol Sci. 2007;18:965-970.

26. Gordon I, Zagoory-Sharon O, Leckman JF, Feldman R. Oxytocin and the development of parenting in humans. Biol Psychiatry. 2010;68: 377-382.

27. Ozsoy S, Esel E, Kula M. Serum oxytocin levels in patients with depression and the effects of gender and antidepressant treatment. Psychiatry Res. 2009;169:249-252.

28. Fasching PA, Faschingbauer F, Goecke TW, et al. Genetic variants in the tryptophan hydroxylase 2 gene (TPH2) and depression during and after pregnancy. J Psychiatr Res. 2012;46(9):1109-1117.

29. Maes M, Verkerk R, Bonaccorso S, Ombelet W, Bosmans E, Scharpé S. Depressive and anxiety symptoms in the early puerperium are related to increased degradation of tryptophan into kynurenine, a phenomenon which is related to immune activation. Life Sci. 2002;71(16):1837-1848.

30. Boufidou F, Lambrinoudaki I, Argeitis J, et al. CSF and plasma cytokines at delivery and postpartum mood disturbances. $J$ Affect Disord. 2009;115(1-2):287-292.

31. Anderson G. Neuronal-immune interactions in mediating stress effects in the etiology and course of schizophrenia: role of the amygdala in developmental co-ordination. Med Hypotheses. 2011;76:54-60.

32. Pocivavsek A, Wu HQ, Elmer GI, Bruno JP, Schwarcz R. Pre- and postnatal exposure to kynurenine causes cognitive deficits in adulthood. Eur J Neurosci. 2012;35(10):1605-1612.

33. Ohira K, Hagihara H, Toyama K, et al. Expression of tryptophan 2,3dioxygenase in mature granule cells of the adult mouse dentate gyrus. Mol Brain. 2010;3:26.

34. Funakoshi H, Kanai M, Nakamura T. Modulation of tryptophan metabolism, promotion of neurogenesis and alteration of anxiety-related behavior in tryptophan 2,3-dioxygenase-deficient mice. Int JTryptophan Res. 2011;4:7-18. 
35. Ren S, Correia MA. Heme: a regulator of rat hepatic tryptophan 2,3-dioxygenase. Arch Biochem Biophys. 2000;377(1):195-203.

36. Laugeray A, Launay JM, Callebert J, Surget A, Belzung C, Barone PR. Peripheral and cerebral metabolic abnormalities of the tryptophankynurenine pathway in a murine model of major depression. Behav Brain Res. 2010;210(1):84-91.

37. Laugeray A, Launay JM, Callebert J, Surget A, Belzung C, Barone PR. Evidence for a key role of the peripheral kynurenine pathway in the modulation of anxiety- and depression-like behaviours in mice: focus on individual differences. Pharmacol Biochem Behav. 2011;98:161-168.

38. Haller J, Mikics E, Halász J, Tóth M. Mechanisms differentiating normal from abnormal aggression: glucocorticoids and serotonin. Eur J Pharmacol. December 5, 2005;526(1-3):89-100.

39. Fuxe K, Jacobsen KX, Höistad M, et al. The dopamine D1 receptorrich main and paracapsular intercalated nerve cell groups of the rat amygdala: relationship to the dopamine innervation. Neuroscience. 2003;119(3):733-746.

40. Love TM, Enoch MA, Hodgkinson CA, et al. Oxytocin gene polymorphisms influence human dopaminergic function in a sex-dependent manner. Biol Psychiatry. 2012;72(3):198-206.

41. Steiner J, Walter M, Gos T, et al. Severe depression is associated with increased microglial quinolinic acid in subregions of the anterior cingulate gyrus: evidence for an immune-modulated glutamatergic neurotransmission? J Neuroinflammation. 2011;8:94.

42. Girdler SS, Klatzkin R. Neurosteroids in the context of stress: implications for depressive disorders. Pharmacol Ther. 2007;116(1):125-139.

43. Brunton PJ, Bales J, Russell JA. Allopregnanolone and induction of endogenous opioid inhibition of oxytocin responses to immune stress in pregnant rats. J Neuroendocrinol. 2012;24(4):690-700.

44. Anderson G, Maes M, Berk M. Inflammation-related disorders in the tryptophan catabolite (TRYCAT) pathway in depression and somatization. Adv Protein Chem Struct Biol. 2012;88:27-48 .

45. Maes M, Rief W. Diagnostic classifications in depression and somatization should include biomarkers, such as disorders in the tryptophan catabolite (TRYCAT) pathway. Psychiatr Res. 2012;196(23):243-249.

46. Anderson G, Maes M, Berk M. Biological underpinnings of the commonalities in depression, somatization, and chronic fatigue syndrome. Med Hypotheses. 2012;78:752-756.

47. Lou HY, Kong JF. The effects of prenatal maternal depressive symptoms on pain scores in the early postpartum period. J Obstet Gynaecol. 2012;32(8):764-766

48. Groer MW, Morgan K. Immune, health and endocrine characteristics of depressed postpartum mothers. Psychoneuroendocrinology. 2007; 32(2):133-139.

49. Meinlschmidt G, Martin C, Neumann ID, Heinrichs M. Maternal cortisol in late pregnancy and hypothalamic-pituitary-adrenal reactivity to psychosocial stress postpartum in women. Stress. 2010;13(2):163-171.

50. Yim IS, Glynn LM, Dunkel-Schetter C, Hobel CJ, Chicz-DeMet A, Sandman CA. Risk of postpartum depressive symptoms with elevated corticotropin-releasing hormone in human pregnancy. Arch Gen Psychiatry. 2009;66(2):162-169.

51. Xie P, Kranzler HR, Poling J, et al. Interaction of FKBP5 with childhood adversity on risk for post-traumatic stress disorder. Neuropsychopharmacology. 2010;35(8):1684-1692.

52. Salel ES, El-Bahei W, El-Hadidy MA, Zayed A. Predictors of postpartum depression in a sample of Egyptian women. Neuropsychiatric Dis Treat. 2013;9:15-24.

53. Newcomer JW, Selke G, Melson AK, Gross J, Vogler GP, Dagogo-Jack S. Dose-dependent cortisol-induced increases in plasma leptin concentration in healthy humans. Arch Gen Psychiatry. 1998;55:995-1000.

54. Luchowska E, Kloc R, Olajossy B, et al. Beta2 adrenergic enhancement of brain kynurenic acid production mediated via cAMP-related protein kinase A signalling. Prog Neuropsychopharmacol Biol Psychiatry. 2009;33(3):519-529.

55. De Rosa V, Procaccini C, Calì G, et al. A key role of leptin in the control of regulatory T cell proliferation. Immunity. 2007;26:241-255.
56. Fukuda M, Williams KW, Gautron L, Elmquist JK. Induction of leptin resistance by activation of cAMP-Epac signaling. Cell Metab. 2011;13: 331-339.

57. Gupta BB, Yanthan L, Singh KM. In vitro effects of 5-hydroxytryptophan, indoleamines and leptin on arylalkylamine $\mathrm{N}$-acetyltransferase (AA-NAT) activity in pineal organ of the fish, Clarias gariepinus (Burchell, 1822) during different phases of the breeding cycle. Indian J Exp Biol. 2010;48(8):786-792.

58. Maes M, Lin AH, Ombelet W, et al. Immune activation in the early puerperium is related to postpartum anxiety and depressive symptoms. Psychoneuroendocrinology. 2000;25(2):121-137.

59. Maes M, Ombelet W, Libbrecht II, et al. Effects of pregnancy and delivery on serum concentrations of Clara Cell Protein (CC16), an endogenous anticytokine: lower serum CC16 is related to postpartum depression. Psychiatry Res. 1999;87(2-3):117-127.

60. Maes M, Ombelet W, De Jongh R, Kenis G, Bosmans E. The inflammatory response following delivery is amplified in women who previously suffered from major depression, suggesting that major depression is accompanied by a sensitization of the inflammatory response system. J Affect Disord. 2001;63(1-3):85-92.

61. Maes M, Bosmans E, Ombelet W. In the puerperium, primiparae exhibit higher levels of anxiety and serum peptidase activity and greater immune responses than multiparae. J Clin Psychiatry. 2004; 65(1):71-76.

62. Maes M, Claes M, Schotte C, et al. Disturbances in dexamethasone suppression test and lower availability of L-tryptophan and tyrosine in early puerperium and in women under contraceptive therapy. J Psychosom Res. 1992;36(2):191-197.

63. Maes M, Ombelet W, Verkerk R, Bosmans E, Scharpé S. Effects of pregnancy and delivery on the availability of plasma tryptophan to the brain: relationships to delivery-induced immune activation and early post-partum anxiety and depression. Psychol Med. 2001;31(5):847-858.

64. Maes M, Ringel K, Kubera M, Berk M, Rybakowski J. Increased autoimmune activity against 5-HT: a key component of depression that is associated with inflammation and activation of cell-mediated immunity, and with severity and staging of depression. JAffect Disord. 2012;136(3):386-392.

65. Donne ML, Settineri S, Benvenga S. Early pospartum alexithymia and risk for depression: relationship with serum thyrotropin, free thyroid hormones and thyroid autoantibodies. Psychoneuroendocrinology. 2012;37(4):519-533.

66. Sylvén SM, Elenis E, Michelakos T, et al. Thyroid function tests at delivery and risk for postpartum depressive symptoms. Psychoneuroendocrinology. In press 2013.

67. Maes M, Ringel, K, Kubera M, et al. In myalgic encephalomyelitis/ chronic fatigue syndrome, increased autoimmune activity against 5-HT is associated with immuno-inflammatory pathways and bacterial translocation. In press 2013

68. Groër M, Davis M, Casey K, Short B, Smith K, Groër S. Neuroendocrine and immune relationships in postpartum fatigue. MCN Am J Matern Child Nurs. 2005;30(2):133-138.

69. Morris G, Maes M. A neuro-immune model of myalgic encephalomyelitis/ chronic fatigue syndrome. Metab Brain Dis. In press 2013.

70. Di Scalea TL, Wisner KL. Antidepressant medication use during breastfeeding. Clin Obstet Gynecol. 2009;52(3):483-497.

71. Nascimento SL, Surita FG, Cecatti JG. Physical exercise during pregnancy: a systematic review. Curr Opin Obstet Gynecol. 2012;24(6): 387-394.

72. Sado M, Ota E, Stickley A, Mori R. Hypnosis during pregnancy, childbirth, and the postnatal period for preventing postnatal depression. Cochrane Database Syst Rev. 2012;6:CD009062.

73. Hübner-Liebermann B, Hausner H, Wittmann M. Recognizing and treating peripartum depression. Dtsch Arztebl Int. 2012;109(24):419-424

74. Ng RC, Hirata CK, Yeung W, Haller E, Finley PR. Pharmacologic treatment for postpartum depression: a systematic review. Pharmacotherapy. 2010;30(9):928-941. 
75. Yonkers KA, Lin H, Howell HB, Heath AC, Cohen LS. Pharmacologic treatment of postpartum women with new-onset major depressive disorder: a randomized controlled trial with paroxetine. $J$ Clin Psychiatry. 2008;69(4):659-665.

76. Appleby L, Warner R, Whitton A, Faragher B. A controlled study of fluoxetine and cognitive-behavioural counselling in the treatment of postnatal depression. BMJ. 1997;314(7085):932-936.

77. Fournier JC, DeRubeis RJ, Hollon SD, et al. Antidepressant drug effects and depression severity: a patient-level meta-analysis. JAMA. 2010;303(1):47-53.

78. Rubinow DR, Schmidt PJ, Roca CA. Estrogen-serotonin interactions: implications for affective regulation. Biol Psychiatry. 1998;44(9): 839-850.

79. Pae CU, Mandelli L, Kim TS, et al. Effectiveness of antidepressant treatments in pre-menopausal versus post-menopausal women: a pilot study on differential effects of sex hormones on antidepressant effects. Biomed Pharmacother. 2009;63(3):228-235.

80. Fortinguerra F, Clavenna A, Bonati M. Psychotropic drug use during breast feeding: a review of the evidence. Pediatrics. 2009;124(4): e547-e556.

81. Weissman A, Levy BT, Hartz AJ, et al. Pooled analysis of antidepressant levels in lactating mothers, breast milk, and nursing infants. Am J Psychiatry. 2004;161(16):1066-1078.

82. Payne JL. Antidepressant use in the postpartum period: practical consideration. Am J Psychiatry. 2007;164(9):1329-1332.

83. Berle JO, Spigset O. Antidepressant use during breastfeeding. Curr Womens Health Rev. 2011;7:28-34.

84. Davanzo R, Copertino M, De Cunto A, Minen F, Amaddeo A. Antidepressant drugs and breastfeeding: a review of the literature. Breastfeed Med. 2011;6(2):89-98.

85. Cutler JA, Rush AJ, McMahon FJ, Laje G. Common genetic variation in the indoleamine-2,3-dioxygenase genes and antidepressant treatment outcome in major depressive disorder. J Psychopharmacol. 2012;26(3): 360-367.

86. Maneeton N, Thongkam A, Maneeton B. Cognitive-behavioral therapy added to fluoxetine in major depressive disorder after 4 weeks of fluoxetine-treatment: 16-week open label study. J Med Assoc Thai. 2010;93(3):337-342.

87. Sarkar S, Gupta R. Telephone vs face-to-face cognitive behavioural therapy for depression. JAMA. 2012;308(11):1090-1091.

88. Bloch M, Schmidt PJ, Danaceau M, et al. Effects of gonadal steroids in women with a history of postpartum depression. Am J Psychiatry. 2000;157(6):924-930.

89. Wjeik J, Dudek D, Schlegel-Zawadzka M, et al. Antepartum/postpartum depressive symptoms and serum zinc and magnesium levels. Pharmacol Rep. 2006;58:571-576.

90. Eby GA, Eby KL. Rapid recovery from major depression using magnesium treatment. Med Hypotheses. 2006;67:362-370.

91. Nakagawasai O, Murata A, Arai Y, et al. Enhanced head-twitch response to 5-HT-related agonists in thiamine. J Neural Transm. 2007;114: $1003-1010$

92. Nikseresht S, Etebary S, Karimian M, Nabavizadeh F, Zarrindast MR, Sadeghipour HR. Acute administration of $\mathrm{Zn}, \mathrm{Mg}$, and thiamine improves postpartum depression conditions in mice. Arch Iran Med. 2012;15(5):306-311.

93. Studd JW. A guide to the treatment of depression in women by estrogens. Climacteric. 2011;14(6):637-642.

94. Ryan J, Scali J, Carrière I, et al. Estrogen receptor alpha gene variants and major depressive episodes. J Affect Disord. 2012;136(3):1222-1226.

95. Anderson $\mathrm{G}$. The role of melatonin in postpartum psychosis and depression associated with bipolar disorder. J Perinat Med. 2010;38(6):585-587.

96. Etain B, Dumaine A, Bellivier F, et al. Genetic and functional abnormalities of the melatonin biosynthesis pathway in patients with bipolar disorder. Hum Mol Genet. 2012;21(18):4030-4037.

97. Galecka E, Szemraj J, Florkowski A, et al. Single nucleotide polymorphisms and mRNA expression for melatonin MT(2) receptor in depression. Psychiatry Res. 2011;189(3):472-474.
98. Parry BL, Meliska CJ, Sorenson DL, et al. Plasma melatonin circadian rhythm disturbances during pregnancy and postpartum in depressed women and women with personal or family histories of depression. Am J Psychiatry. 2008;165(12):1551-1558.

99. Hardeland R, Cardinali DP, Srinivasan V, Spence DW, Brown GM, Pandi-Perumal SR. Melatonin - a pleiotropic, orchestrating regulator molecule. Prog Neurobiol. 2011;93:350-384.

100. Lanoix D, Beghdadi H, Lafond J, Vaillancourt C. Human placental trophoblasts synthesize melatonin and express its receptors. J Pineal Res. 2008;45(1):50-60.

101. Gitto E, Pellegrino S, Gitto P, Barberi I, Reiter RJ. Oxidative stress of the newborn in the pre- and postnatal period and the clinical utility of melatonin. J Pineal Res. 2009;46:128-139.

102. Cohen Engler A, Hadash A, Shehadeh N, Pillar G. Breastfeeding may improve nocturnal sleep and reduce infantile colic: potential role of breast milk melatonin. Eur J Pediatr. 2012;171(4):729-732.

103. Pontes GN, Cardoso EC, Carneiro-Sampaio MM, Markus RP. Pineal melatonin and the innate immune response: the TNF-alpha increase after cesarean section suppress nocturnal melatonin production. $J$ Pineal Res. 2007;43:365-371.

104. Wloch C, Wilson J, Lamagni T, Harrington P, Charlett A, Sheridan E. Risk factors for surgical site infection following caesarean section in England: results from a multicentre cohort study. BJOG. 2012;119(11): 1324-3133.

105. Eisenach JC, Pan PH, Smiley R, Lavand'homme P, Landau R, Houle TT. Severity of acute pain after childbirth, but not type of delivery, predicts persistent pain and postpartum depression. Pain. 2008;140(1):87-94.

106. Andreasen JT, Redrobe JP, Nielsen EØ. Combined $\alpha 7$ nicotinic acetylcholine receptor agonism and partial serotonin transporter inhibition produce antidepressant-like effects in the mouse forced swim and tail suspension tests: a comparison of SSR180711 and PNU-282987. Pharmacol Biochem Behav. 2012;100(3):624-629.

107. Fernandez JW, Grizzell JA, Wecker L. The role of estrogen receptor $\beta$ and nicotinic cholinergic receptors in postpartum depression. Prog Neuropsychopharmacol Biol Psychiatry. 2012;40C:199-206.

108. Cosi C, Mannaioni G, Cozzi A, et al. G-protein coupled receptor 35 (GPR35) activation and inflammatory pain: Studies on the antinociceptive effects of kynurenic acid and zaprinast. Neuropharmacology. 2011;60:1227-1231.

109. Markus RP, Silva CLM, Franco DG, Barbosa EM Jr, Ferreira ZS. Is modulation of nicotinic acetylcholine receptors by melatonin relevant for therapy with cholinergic drugs? Pharmacol Therapeut. 2010;126: 251-262.

110. Bennet S, Alpert M, Kubulins V, Hansler RL. Use of modified spectacles and light bulbs to block blue light at night may prevent postpartum depression. Med Hypotheses. 2009;73(2):251-253.

111. Sharkey JT, Cable C, Olcese J. Melatonin sensitizes human myometrial cells to oxytocin in a protein kinase $\mathrm{C}$ alpha/extracellular-signal regulated kinase-dependent manner. J Clin Endocrinol Metab. 2010;95(6):2902-2908.

112. Juszczak M, Boczek-Leszczyk E. Hypothalamic gonadotropinreleasing hormone receptor activation stimulates oxytocin release from the rat hypothalamo-neurohypophysial system while melatonin inhibits this process. Brain Res Bull. 2010;81(1):185-190.

113. Labuschagne I, Phan KL, Wood A, et al. Oxytocin attenuates amygdala reactivity to fear in generalized social anxiety disorder. Neuropsychopharmacology. 2010;35:2403-2413.

114. Heinrichs M, Baumgartner T, Kirschbaum C, Ehlert U. Social support and oxytocin interact to suppress cortisol and subjective responses to psychosocial stress. Biol Psychiatry. 2003;54:1389-1398.

115. Srinivasan V, Zakaria R, Othman Z, Lauterbach EC, AcuñaCastroviejo D. Agomelatine in depressive disorders: its novel mechanisms of action. J Neuropsychiatry Clin Neurosci. 2012;24(3): 290-308. 
Neuropsychiatric Disease and Treatment

Dovepress

\section{Publish your work in this journal}

Neuropsychiatric Disease and Treatment is an international, peerreviewed journal of clinical therapeutics and pharmacology focusing on concise rapid reporting of clinical or pre-clinical studies on a range of neuropsychiatric and neurological disorders. This journa is indexed on PubMed Central, the 'PsycINFO' database and CAS.

The manuscript management system is completely online and includes a very quick and fair peer-review system, which is all easy to use. Visit http://www.dovepress.com/testimonials.php to read real quotes from published authors.

Submit your manuscript here: http://www.dovepress.com/neuropsychiatric-disease-and-treatment-journal 